\title{
IncRNA LINC01535 upregulates BMP2 expression levels to promote osteogenic differentiation via sponging miR-3619-5p
}

\author{
YIWEN ZHAO, YI CHEN, XINYU HU, NAIDONG ZHANG and FENG WANG \\ Department of Osteological Surgery, The Third Affiliated Hospital of Soochow University, \\ Changzhou, Jiangsu 213000, P.R. China
}

Received November 26, 2019; Accepted August 20, 2020

DOI: $10.3892 / \mathrm{mmr} .2020 .11635$

\begin{abstract}
Osteoporosis is a debilitating skeletal disease that causes bones to collapse and is accompanied by a high risk of bone fracture. It was previously demonstrated that the osteogenic differentiation of human bone marrow-derived mesenchymal stem cells (hBMSCs) serves an important role in the process of human bone formation. Accumulating research has indicated that long non-coding RNAs (lncRNAs) participate in hBMSC osteogenic differentiation. For example, LINC01535 was reported to serve as a carcinogenic factor in cervical cancer; however, its latent function and molecular mechanism in the osteogenesis of hBMSCs remain to be investigated. The present study showed that the expression levels of LINC01535 were upregulated upon increasing osteogenic differentiation time. In addition, the inhibition of LINC01535 inhibited hBMSC proliferation and osteogenic differentiation and promoted cell apoptosis. Using bioinformatics analysis, LINC01535 was discovered to have complementary binding sites for microRNA (miR)-3619-5p, and further experiments demonstrated that LINC01535 functioned as a sponge of miR-3619-5p. Additionally, bone morphogenetic protein 2 (BMP2) was confirmed to be a target of miR-3619-5p. The results revealed that LINC01535 regulated the expression levels of BMP2 via sponging miR-3619-5p. In conclusion, the findings of the present study suggested that LINC01535 may accelerate the osteogenic process of hBMSCs via targeting the miR-3619-5p/BMP2 axis, which may offer an innovative therapeutic method for osteoporosis.
\end{abstract}

Correspondence to: Dr Feng Wang, Department of Osteological Surgery, The Third Affiliated Hospital of Soochow University, 185 Juqian Street, Changzhou, Jiangsu 213000, P.R. China E-mail: biggestbee@163.com

Key words: osteoporosis, LINC01535, human bone marrow-derived mesenchymal stem cells, microRNA-3619-5p, bone morphogenetic protein 2

\section{Introduction}

Osteoporosis is a widespread skeletal disorder resulting from imbalances in bone homeostasis $(1,2)$. Osteoporosis is a critical contributor of mortality in the elderly and is a socioeconomic challenge in terms of public health (3). The incidence of osteoporosis was $27.96 \%$ between 2012 and 2015 in China (4). During the process of bone remodeling, osteoblasts generate new bone tissues, while osteoclasts contribute to the destruction and reabsorption of bone tissues (5). Bone marrow-derived mesenchymal stem cells (BMSCs) belong to a class of pluripotent stem cells, which are characterized by their ability to differentiate into numerous cell types, including chondrocytes, osteoblasts and adipocytes $(6,7)$. Calcium and vitamin D are the basic treatment for osteoporosis (8). Considering that osteoblasts serve a pivotal role in the dynamic balance between bone formation and bone resorption (9), determining the latent mechanism regulating the osteogenic differentiation of BMSCs is required to improve the therapeutic treatment of osteoporosis.

Long non-coding RNAs (lncRNAs) are a type of non-coding RNAs $>200$ nucleotides in length that lack protein-coding capacity $(10,11)$. IncRNAs have attracted extensive attention and were reported to be involved in the initiation and evolution of numerous types of disease, such as atherosclerosis, inflammatory bowel disease and cancer (12-14). Accumulating evidence has identified that lncRNAs serve as vital modulators in a wide spectrum of biological activities, including cell differentiation (15). Studies have also demonstrated that lncRNAs are vital in the development of several orthopedic disorders, such as osteoporosis and ankylosis spondylitis $(16,17)$, through their regulation in osteogenesis. For instance, lncRNA metastasis associated lung adenocarcinoma transcript 1 impeded the osteogenic differentiation of mesenchymal stem cells in osteoporosis model rats via the MAPK signaling pathway (18); overexpression of lncRNA maternally expressed gene 3 promoted the osteogenic differentiation of mesenchymal stem cells of patients with multiple myeloma via activating the transcription of bone morphogenetic protein (BMP) 4 (19) and the lncRNA HOX transcript antisense RNA suppressed microRNA (miRNA/miR)-17-5p expression, which affected mesenchymal stem cell osteogenic differentiation and proliferation in non-traumatic osteonecrosis of the femoral head (20). However, although LINC01535 has been reported 
to exert oncogenic activities in cervical cancer (21), to the best of our knowledge, the biological function of LINC01535 in osteogenesis remains elusive.

miRNAs are a category of small non-coding RNAs that are 18-22 nucleotides in length (22). miRNAs regulate the expression levels of target genes through binding with their 3'-untranslated regions (3'-UTRs) to either inhibit their degradation or translation $(23,24)$. miRNAs may be implicated in the pathogenesis of osteoporosis via regulating genes closely associated with bone homeostatic processes at the post-transcriptional level (25). However, despite the reported tumor suppressive properties of miR-3619-5p in human cancers (26), its role and regulatory mechanism in osteogenic differentiation remains poorly understood. BMP2 belongs to the TGF- $\beta$ superfamily, where it serves a crucial role in bone regeneration through regulating the differentiation of osteoblast progenitors or stem cells $(27,28)$. Previous studies have confirmed that numerous miRNAs affect the progression of osteogenesis via targeting BMP2 (29-31). Therefore, the present study aimed to investigate the relationship between miR-3619-5p and BMP2.

The current study hypothesized that LINC01535 may be involved in the process of BMSC osteogenic differentiation. The findings revealed that LINC01535 contributed to the differentiation of BMSCs by targeting the miR-3619-5p/BMP2 axis.

\section{Materials and methods}

Cell culture. Human BMSCs (hBMSCs) were obtained from the American Type Culture Collection. hBMSCs were cultured in DMEM (HyClone; Cytiva), supplemented with 10\% FBS (Gibco; Thermo Fisher Scientific, Inc.), 100 mg/l streptomycin and $100 \mathrm{U} / 1$ penicillin, and maintained at $37^{\circ} \mathrm{C}$ in the presence of $5 \% \mathrm{CO}_{2}$. To induce osteogenic differentiation, hBMSCs were maintained in osteogenic supplement (OS) medium (Sigma-Aldrich; Merck KGaA) with $10 \mathrm{mM}$ $\beta$-glycerophosphate (Sigma-Aldrich; Merck KGaA), $100 \mathrm{nM}$ dexamethasone (Sigma-Aldrich; Merck KGaA) and $100 \mathrm{nM}$ ascorbic acid (Sigma-Aldrich; Merck KGaA) for 14 days. The OS medium was replaced every 2 days.

Cell transfection. A short hairpin RNA (shRNA) vector containing cDNA oligonucleotides targeting LINC01535 (sh-LINC01535; Shanghai GenePharma Co., Ltd.) was used to knock down LINC01535 expression levels. BMP2-specific shRNA (sh-BMP2; Invitrogen; Thermo Fisher Scientific, Inc.) was used to knock down BMP2 expression levels. Scramble shRNAs (sh-NC; Shanghai GenePharma Co., Ltd.) were used as the negative controls (NCs). miR-3619-5p mimic (5'-AAG CGAGACAGTAGCATGCGGA-3'), miR-3619-5p inhibitor (5'-TCAGCTTGCTACTGTSTCCGTT-3') and their respective NCs (NC mimic, 5'-AATGAGGCGTGAGATGGAGGCT-3' and NC inhibitor, 5'-GACCTGGATCTCAGCCTAGCTT-3') were designed and generated by Guangzhou RiboBio Co., Ltd. To explore whether LINC01535 facilitated the osteogenesis of hBMSCs via the miR-3619-5p/BMP2 axis, hBMSCs were transfected with sh-NC + NC inhibitor, sh-LINC01535 + NC inhibitor, sh-LINC01535 + miR-3619 inhibitor or sh-LINC01535 + miR-3619 inhibitor + sh-BMP2. hBMSCs were transfected with the indicated plasmids $(10 \mathrm{nM})$ using Lipofectamine ${ }^{\circledR} 3000$ (Invitrogen; Thermo Fisher Scientific, Inc.), according to the manufacturer's protocol. Subsequent experiments were performed $48 \mathrm{~h}$ post-transfection.

Reverse transcription-quantitative PCR (RT-qPCR). Total RNA was extracted from hBMSCs using TRIzol ${ }^{\circledR}$ reagent (Invitrogen; Thermo Fisher Scientific, Inc.), according to the manufacturer's protocol. Total RNA was reverse transcribed into cDNA using a SuperScript III kit (Invitrogen; Thermo Fisher Scientific, Inc.), according to the manufacturer's instructions. qPCR was subsequently performed on an ABI 7500 Fast Real-Time PCR system (Applied Biosystems; Thermo Fisher Scientific, Inc.) using SYBR Green Master mix (Thermo Fisher Scientific, Inc.). The following thermocycling conditions were used for qPCR: Initial denaturation at $95^{\circ} \mathrm{C}$ for $3 \mathrm{~min}, 40$ cycles of $95^{\circ} \mathrm{C}$ for $5 \mathrm{sec}$ and $60^{\circ} \mathrm{C}$ for $30 \mathrm{sec}$. The following primer pairs were used for the qPCR: LINC01535 forward, 5'-GGGATGGAAGTGTGATTGC-3' and reverse, 5'-TGATGCTAGGGGTGCTAAG-3'; miR-3619-5p forward, 5'-TCAGCAGGCAGGCTGGTGC-3' and reverse, 5'-GAA CATGTCTGCGTATCTC-3'; BMP2 forward, 5'-AAGCGT CAAGCCAAACACAAAC-3' and reverse, 5'-GCCACGATC CAGTCATTCCAC-3'; osteocalcin (OCN) forward, 5'-GCC GAGAAATGTTGGAGAAA-3' and reverse, 5'-CTCCTTAAT CTGGCCAACCA-3'; osterix (OSX) forward, 5'-CAGGCT ATGCTAATGATTACC-3' and reverse, 5'-GGCAGACAG TCAGAAGAG-3'; runt-related transcription factor 2 (RUNX2) forward, 5'-CGAATAACAGCACGCTATTAA-3' and reverse, 5'-GTCGCCAAACAGATTCATCCA-3'; GAPDH forward, 5'-AGGTCGGTGTGAACGGATTTG-3' and reverse, 5'-TGT AGACCATGTAGTTGAGGTCA-3' and U6 forward, 5'-CGC TTCG GCAGCACATATACTA-3' and reverse, 5'-CGCTTC ACGAATTTGCGTGTCA-3'. GAPDH and U6 were used as the endogenous loading controls. The $2^{-\Delta \Delta \mathrm{Cq}}$ method (32) was used to quantify gene expression levels.

Cell counting Kit-8 (CCK-8) assay. Cell proliferation was analyzed using a CCK- 8 assay (Dojindo Molecular Technologies, Inc.) according to the manufacturer's protocol. Following transfection, hBMSCs were plated into a 96-well plate at a density of $1 \times 10^{4}$ cells/well. A total of $10 \mu \mathrm{CCK}-8$ solution was added per well and incubated for $4 \mathrm{~h}$ at $37^{\circ} \mathrm{C}$. Subsequently, the optical density value was determined at a wavelength of $450 \mathrm{~nm}$ using a microplate reader (Bio-Rad Laboratories, Inc.).

Alkaline phosphatase (ALP) activity measurement. An ALP activity assay was performed using an ALP detection kit (cat. no. A-059-2; Nanjing Jiancheng Bioengineering Institute) according to the manufacturer's protocol. Briefly, following osteogenic induction, hBMSCs were seeded into 96-well plates at 6,000 cells per well, and subsequently treated with $50 \mu \mathrm{l}$ buffer solution and $50 \mu \mathrm{l}$ substrate solution. Following incubation for $15 \mathrm{~min}$ at $37^{\circ} \mathrm{C}$, each well was supplemented with $150 \mu \mathrm{l}$ color-developing reagent and the absorbance was determined at a wavelength of $520 \mathrm{~nm}$ using a microplate reader (Thermo Fisher Scientific, Inc.). ALP activity was presented as King units $/ 100 \mathrm{ml}$. All experiments were repeated three times. 
Western blotting. Total protein was extracted from hBMSCs using RIPA lysis buffer (Beyotime Institute of Biotechnology) containing protease inhibitors (Roche Diagnostics). Total protein was quantified using a BCA Protein assay kit (Thermo Fisher Scientific, Inc.), according to the manufacturer's instructions. A total of $10 \mu \mathrm{g}$ protein/lane was separated via $10 \%$ SDS-PAGE. The separated proteins were subsequently transferred onto PVDF membranes and blocked with $5 \%$ non-fat milk for $2 \mathrm{~h}$ at room temperature. The membranes were then incubated overnight with primary antibodies at $4{ }^{\circ} \mathrm{C}$. Following primary antibody incubation, the PVDF membranes were incubated with horseradish peroxidase-conjugated goat anti-mouse IgG (cat. no. ab205719) and goat anti-rabbit IgG (cat. no. ab205718) secondary antibodies (1:1,000; both purchased form Abcam) for $2 \mathrm{~h}$ at room temperature. Total protein was visualized using an enhanced chemiluminescence kit (EMD Millipore) and semi-quantified using Quantity One software (version 4.6.9; Bio-Rad Laboratories, Inc.). The following primary antibodies were used: Anti-OCN (1:1,000; cat.no.ab93876; Abcam), anti-OSX (1:1,000; cat.no. ab209484; Abcam), anti-RUNX2 (1:1,000; cat. no. 12556; Cell Signaling Technology, Inc.), anti-BMP2 (1:1,000; cat. no. ab214821; Abcam) and anti-GAPDH (1:1,000; cat. no. ab8245; Abcam). GAPDH was used as the loading control.

RNA immunoprecipitation (RIP) assay. The EZMagna RIP kit (EMD Millipore) was used to perform RIP experiments according to the manufacturer's protocol. Briefly, hBMSCs were dissolved in lysis buffer and centrifuged at $10,000 \mathrm{x}$ g for $10 \mathrm{~min}$ at $4^{\circ} \mathrm{C}$. The cell lysates were added to the RIP buffer, which was supplemented with magnetic beads coated with anti-protein argonaute-2 (Ago2; cat. no. MABE253; EMD Millipore) antibody and negative control anti-IgG antibody (cat. no. AB22-K; EMD Millipore) and incubated overnight at $4^{\circ} \mathrm{C}$. Samples were then treated with proteinase $\mathrm{K}$. Following elution from the beads, the immunoprecipitated RNA was purified and subjected to RT-qPCR analysis.

Luciferase reporter assay. StarBase 2.0 (http://starbase. sysu.edu.cn) was used to predict the downstream target of LINC01535 and miR-3619-5p. The 3'-UTR sequences of LINC01535 harboring miR-3619-5p binding sites were cloned into the pmirGLO luciferase plasmid (Promega Corporation) to construct LINC01535-wild-type (WT). To construct LINC01535-mutant (Mut) vectors, miR-3619-5p binding sites were mutated. Following the same protocol, the WT and Mut plasmids of BMP2 were generated. hBMSCs were co-transfected with the corresponding luciferase vectors and miR-3619-5p mimic or NC mimic using Lipofectamine 3000 (Invitrogen; Thermo Fisher Scientific, Inc.). Following $48 \mathrm{~h}$ of incubation, the hBMSCs were collected and the relative luciferase activity was analyzed using a Dual-Luciferase Reporter Assay system (Promega Corporation) according to the manufacturer's protocol and normalized to Renilla luciferase activity.

Statistical analysis. Experimental data are presented as the mean \pm SD and all assays were performed in triplicate. Statistical analysis was performed using SPSS 21.0 software (IBM Corp.). Statistical differences between two groups were determined using Student's t-test. Statistical differences among $\geq 2$ groups was determined using one-way ANOVA followed by Tukey's post hoc test. $\mathrm{P}<0.05$ was considered to indicate a statistically significant difference.

\section{Results}

LINC01535 expression levels are markedly upregulated during osteogenic induction. To stimulate the osteogenic differentiation of hBMSCs, hBMSCs were treated with OS medium for 14 days. The results revealed that the expression levels of osteogenesis-related genes, OCN, OSX and RUNX2, were gradually upregulated as the osteogenic induction time increased (Fig. 1A). Similarly, western blotting demonstrated that the protein expression levels of OCN, OSX and RUNX2 were also markedly upregulated following treatment with OS medium (Fig. 1B). The ALP activity assay also showed that the administration of OS medium significantly enhanced the ALP activity of hBMSCs (Fig. 1C). The CCK-8 assay revealed that the cell viability of hBMSCs gradually increased during the process of osteogenic differentiation (Fig. 1D). RT-qPCR analysis illustrated that LINC01535 expression levels were upregulated during the process of osteoblast differentiation (Fig. 1E). Altogether, these findings indicated that LINC01535 may be upregulated during osteogenic induction.

Silencing of LINC01535 inhibits the osteogenic differentiation and proliferation of hBMSCs. The present study subsequently aimed to investigate the role of LINC01535 in osteogenesis. The knockdown transfection efficiency of LINC01535 was verified using a RT-qPCR assay (Fig. 2A) and loss-of-function experiments were then performed. The mRNA and protein expression levels of OCN, OSX and RUNX2 were markedly downregulated following LINC01535 knockdown (Fig. 2B and C). In addition, the effects of LINC01535 knockdown on osteogenic differentiation were assessed using an ALP activity assay and the results indicated that LINC01535 knockdown decreased ALP activity (Fig. 2D). Moreover, the CCK-8 assay demonstrated that the silencing of LINC01535 significantly inhibited hBMSC proliferation at $72 \mathrm{~h}$ (Fig. 2E). Altogether, these results indicated that LINC01535 knockdown may inhibit hBMSC osteogenic differentiation and proliferation.

LINC01535 functions as a sponge for miR-3619-5p. Using bioinformatics analysis, miR-3619-5p was predicted to have complementary binding sites for LINC01535 (Fig. 3A). To further verify the relationship between miR-3619-5p and LINC01535, a dual-luciferase reporter assay was performed. Firstly, RT-qPCR demonstrated that miR-3619 expression was significantly upregulated in hBMSCs transfected with miR-3619 mimic compared with NC mimic-transfected cells (Fig. 3B). Furthermore, the dual-luciferase reporter assay revealed that miR-3619-5p overexpression significantly decreased the relative luciferase activity of the LINC01535-WT vector. Meanwhile, no significant differences were observed in the relative luciferase activity of the LINC01535-Mut vector, thus validating the interaction between LINC01535 and miR-3619-5p (Fig. 3C). In addition, RT-qPCR analysis 
A

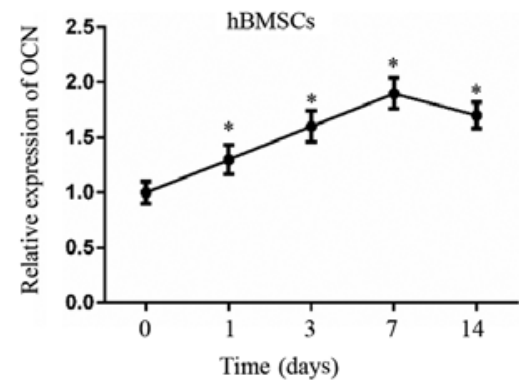

B

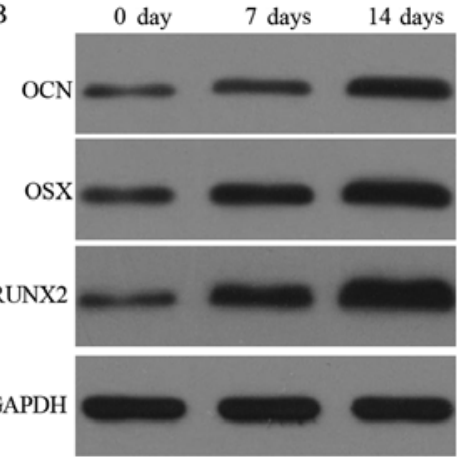

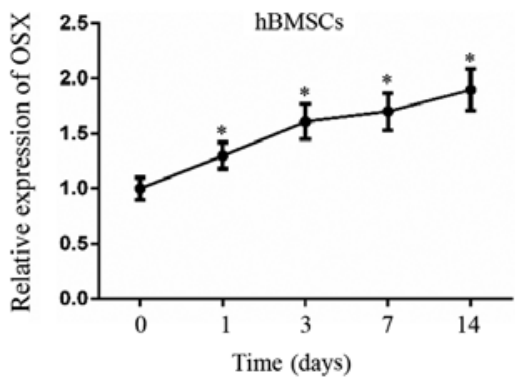

$\mathrm{D}$

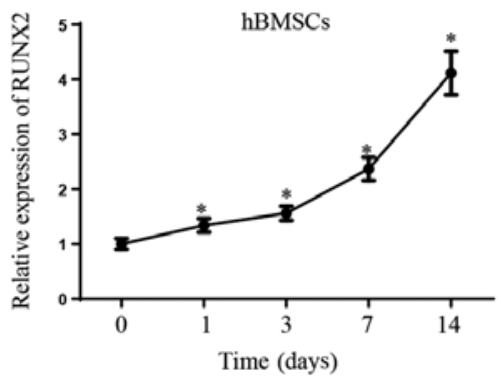

E
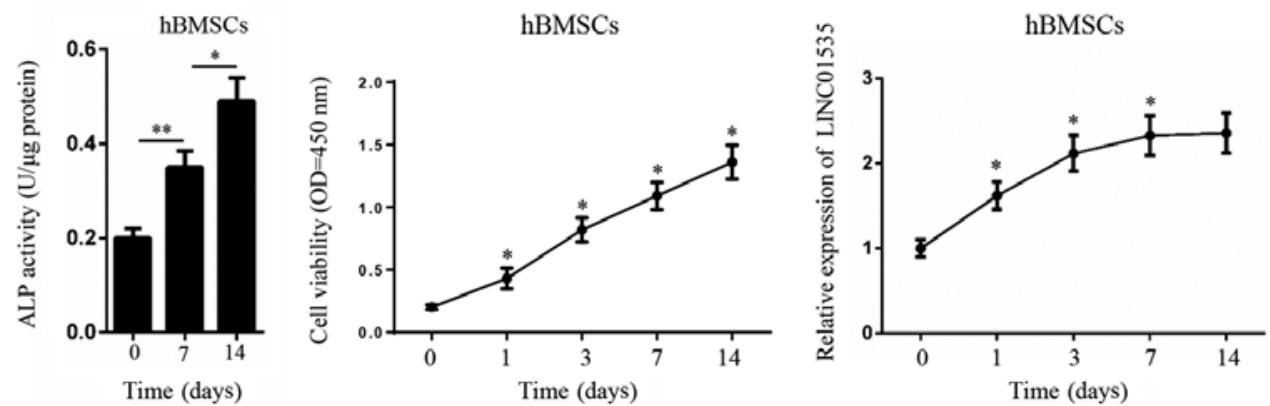

Figure 1. LINC01535 expression is increased during osteogenic induction. (A) RT-qPCR assay was performed to assess the mRNA expression of OCN, OSX and RUNX2 in hBMSCs at 0, 1, 3, 7 and 14 days-post osteogenic induction. (B) Western blotting was performed to assess the protein levels of OCN, OSX and RUNX2 in hBMSCs during osteogenic induction. (C) ALP activity assay was performed to estimate osteogenic differentiation of hBMSCs. (D) Cell Counting Kit- 8 assay showed the cell viability of hBMSCs during the process of osteogenic differentiation. (E) LINC01535 expression in hBMSCs after treatment with OS medium as measured by RT-qPCR. "P<0.05 and ${ }^{* *} \mathrm{P}<0.01$ vs. 0 day group or as indicated. OCN, osteocalcin; OSX, osterix; RUNX2, runt-related transcription factor 2; hBMSCs, human bone marrow-derived mesenchymal stem cells; ALP, alkaline phosphatase; RT-qPCR, reverse transcription-quantitative PCR; OD, optical density.
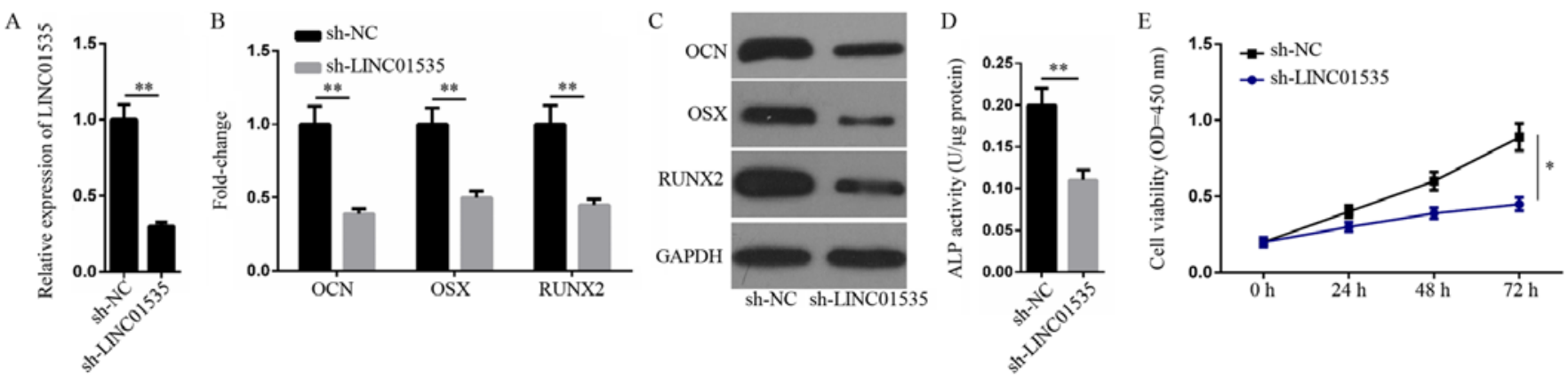

Figure 2. Silencing of LINC01535 inhibits the osteogenic differentiation and proliferation of hBMSCs. (A) Following transfection with sh-NC or sh-LINC01535, RT-qPCR analysis was performed to assess the transfection efficiency of sh-LINC01535. The expression of OCN, OSX and RUNX2 at (B) mRNA and (C) protein levels in hBMSCs was examined by RT-qPCR and western blotting, respectively, in LINC01535 knockdown hBMSCs. (D) The function of LINC01535 in hBMSC osteogenic differentiation was evaluated by detecting ALP activity. (E) The cell viability in LINC01535-knockdown hBMSCs was assessed using a Cell Counting Kit-8 assay. ${ }^{*} \mathrm{P}<0.05$ and ${ }^{* * *} \mathrm{P}<0.01$. OCN, osteocalcin; OSX, osterix; RUNX2, runt-related transcription factor 2; hBMSCs, human bone marrow-derived mesenchymal stem cells; sh, short hairpin RNA; NC, negative control; OD, optical density; RT-qPCR, reverse transcription-quantitative PCR; ALP, alkaline phosphatase.

demonstrated that the silencing of LINC01535 significantly upregulated miR-3619-5p expression levels compared with the sh-NC group (Fig. 3D). Simultaneously, the upregulated expression levels of miR-3619-5p resulted in the downregulated expression levels of LINC01535 (Fig. 3E). Altogether, these findings suggested that LINC01535 may interact with miR-3619-5p by direct binding.

LINC01535 promotes BMP2 expression levels by competing for $m i R-3619-5 p$. The starBase website was used to identify target genes of miR-3619-5p. The analysis identified a complementary binding site between BMP2 and miR-3619-5p (Fig. 4A). A luciferase reporter gene assay further revealed that the relative luciferase activity of the BMP2-WT vector significantly decreased when co-transfected with the miR-3619-5p mimic compared with the NC mimic, whereas the co-transfection with the NC mimic did not affect relative luciferase activity (Fig. 4B). In addition, the results demonstrated that the upregulation of miR-3619-5p expression levels markedly downregulated the BMP2 expression at both mRNA and protein levels (Fig. 4C and D). RIP assay results illustrated that LINC01535, miR-3619-5p and BMP2 were all abundantly 
A

Mutant LINC01535: 5' CUCCUGGGUUAAGAACAU 3'
Wild-type LINC01535: 5' CUCCUGGGUUCCUGCUGU 3'

hsa-miR-3619-5p: 3' GUGGUCGGACGGACGACU 5'
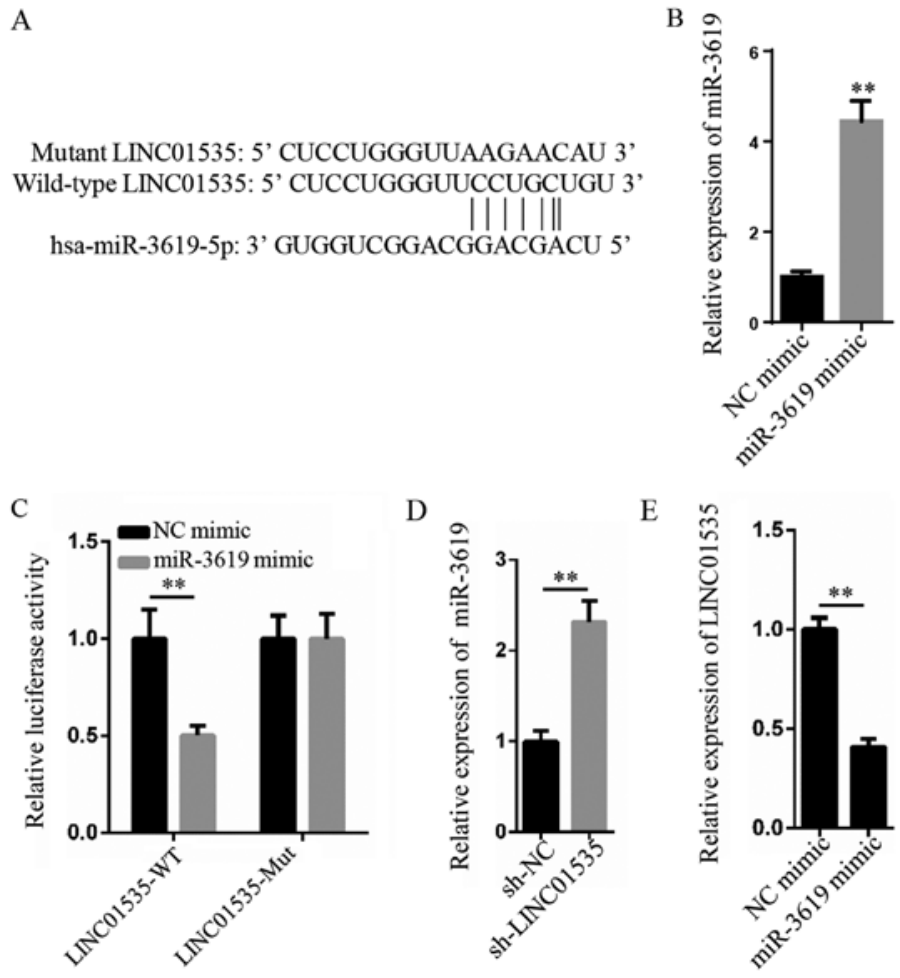

Figure 3. LINC01535 functions as a sponge for miR-3619-5p. (A) The putative binding sites between LINC01535 and miR-3619-5p. (B) RT-qPCR showing the relative expression of miR-3619-5p in hBMSCs transfected with NC mimic and miR-3619 mimic. (C) The binding specificity of LINC01535 to miR-3619-5p was confirmed by a luciferase reporter assay. (D) RT-qPCR detection of LINC01535 expression in hBMSCs transfected with sh-NC or sh-LINC01535. (E) The levels of LINC01535 in hBMSCs were measured by RT-qPCR in miR-3619-5p-overexpressing hBMSCs. ${ }^{* *} \mathrm{P}<0.01$. miR, microRNA; RT-qPCR, reverse transcription-quantitative PCR; NC, negative control; sh, short hairpin RNA; hBMSCs, human bone marrow-derived mesenchymal stem cells; WT, wild-type; Mut, mutant.

A

Mutant BMP2: 5' AGUGGAUAAUCCACUACAACA 3' Wild-type BMP2: 5' AGUGGAUAAUCCACUCUGCUG 3' hsa-miR-3619-5p: 3' CGACGUGGUCGGACGGACGAC 5'
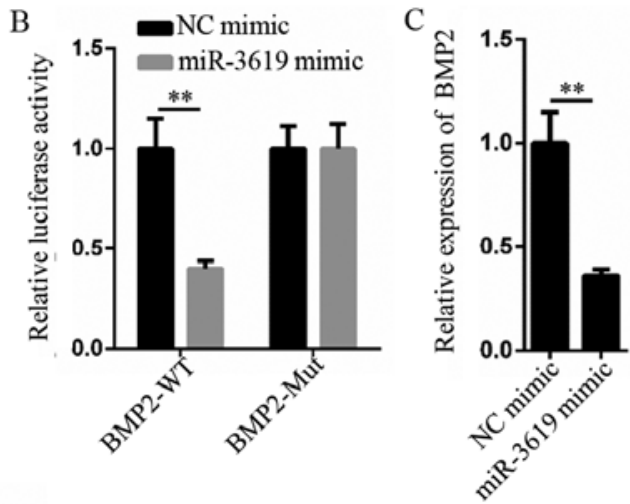

D

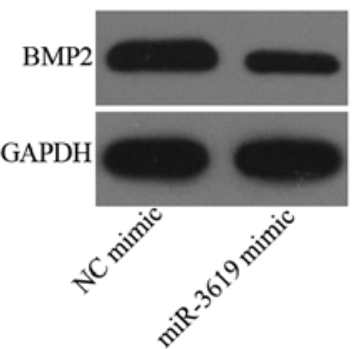

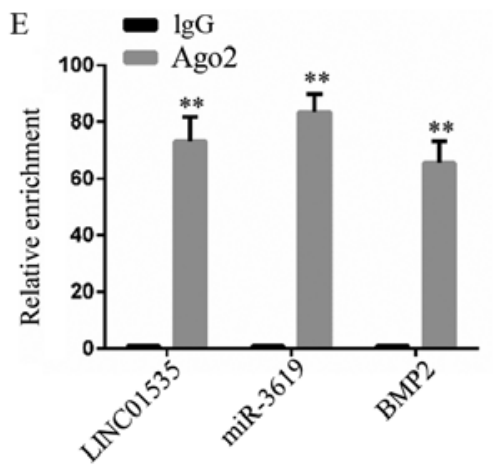

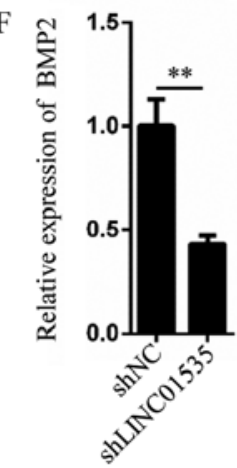

G

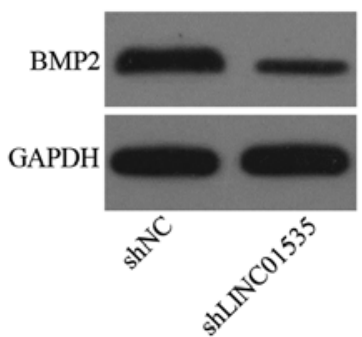

Figure 4. LINC01535 promotes BMP2 expression by competing for miR-3619-5p. (A) The potential miR-3619-5p binding sites in the 3'-untranslated region of BMP2. (B) Luciferase reporter assay was performed to validate whether BMP2 directly binds to miR-3619-5p. (C) mRNA and (D) protein expression of BMP2 in human bone marrow-derived mesenchymal stem cells transfected with NC mimic or miR-3619-5p mimic as assessed by RT-qPCR and western blotting. (E) RNA immunoprecipitation assay was performed to verify the interaction between LINC01535, miR-3619-5p and BMP2. RT-qPCR and western blot were used to assess the effects of LINC01535 depletion on BMP2 (F) mRNA and (G) protein levels. ${ }^{* *} \mathrm{P}<0.01 \mathrm{vs.} \operatorname{lgG}$ or as indicated. miR, microRNA; RT-qPCR, reverse transcription-quantitative PCR; NC, negative control; Ago2, protein argonaute-2; BMP2, bone morphogenetic protein 2; WT, wild-type; Mut, mutant. 

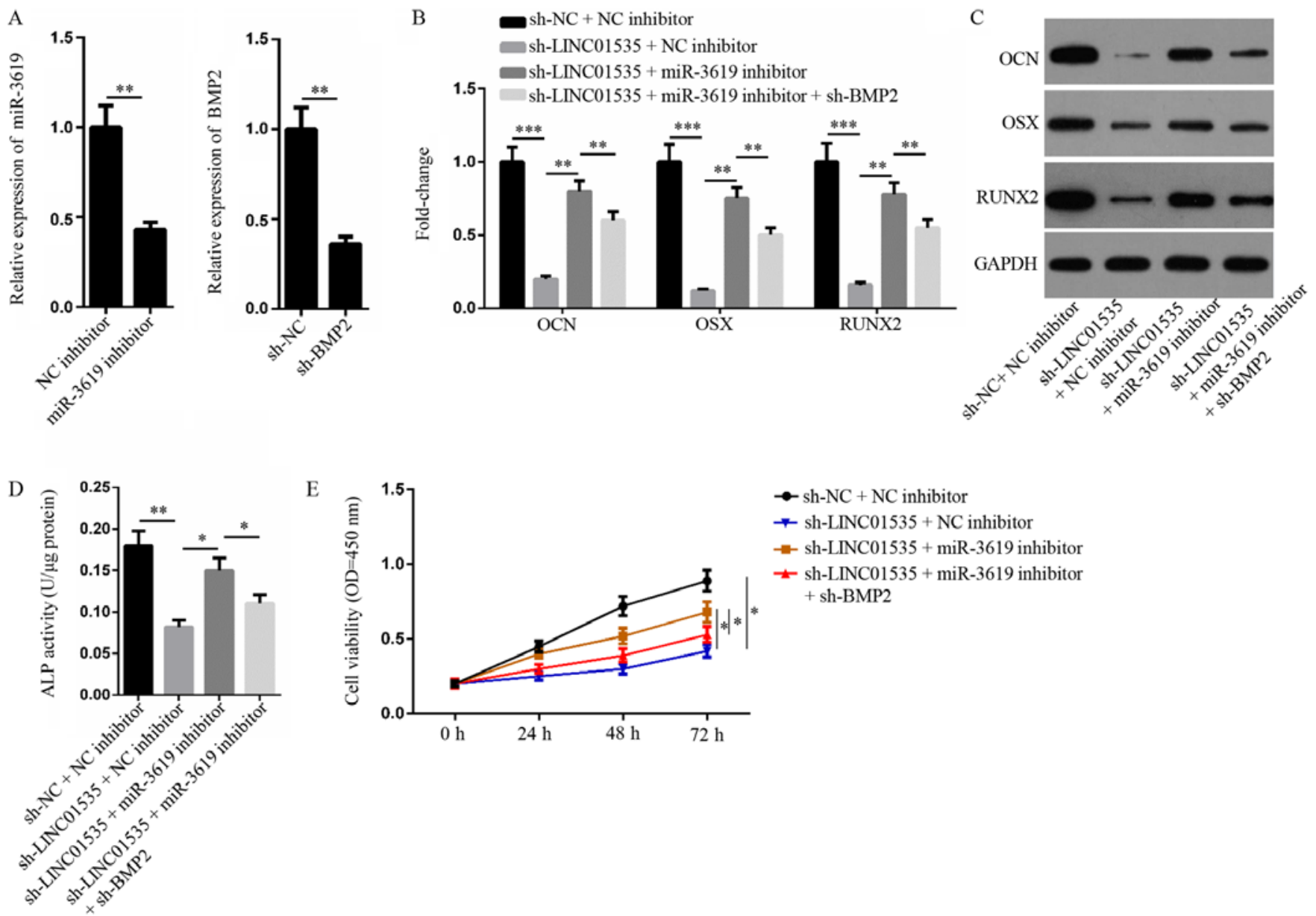

Figure 5. LINC01535 facilitates the osteogenesis of hBMSCs via the miR-3619-5p/BMP2 axis. To assess the role of LINC01535/miR-3619-5p/BMP2 in hBMSC osteogenesis, hBMSCs were transfected with different vectors to generate four groups: sh-NC + NC inhibitor, sh-LINC01535 + NC inhibitor, sh-LINC01535 + miR-3619-5p inhibitor and sh-LINC01535 + miR-3619-5p inhibitor + sh-BMP2. (A) The inhibition efficiency of miR-3619-5p and sh-BMP2 was examined by RT-qPCR. The (B) mRNA and (C) protein levels of OCN, OSX and RUNX2 in the four groups were measured by RT-qPCR and western blotting, respectively. (D) The osteogenic differentiation of hBMSCs in described groups was evaluated by ALP activity detection. (E) Cell Counting Kit-8 assay was performed to assess hBMSC proliferation following transfection with indicated vectors and reagents. ${ }^{*} \mathrm{P}<0.05,{ }^{* * *} \mathrm{P}<0.01$ and ${ }^{* * *} \mathrm{P}<0.005$. miR, microRNA; RT-qPCR, reverse transcription-quantitative PCR; NC, negative control; BMP2, bone morphogenetic protein 2; OCN, osteocalcin; OSX, osterix; RUNX2, runt-related transcription factor 2; hBMSCs, human bone marrow-derived mesenchymal stem cells; sh, short hairpin RNA; ALP, alkaline phosphatase.

expressed in the complexes precipitated by the anti-Ago2 antibody (Fig. 4E). In addition, LINC01535 decreased the mRNA and protein expression levels of BMP2 compared with the sh-NC group (Fig. 4F and G). Therefore, based on the aforementioned findings, it was hypothesized that LINC01535 may sponge miR-3619-5p to upregulate the BMP2 expression levels.

LINC01535 facilitates the osteogenesis of hBMSCs via the $m i R-3619-5 p / B M P 2$ axis. Due to the competing nature of LINC01535 over miR-3619-5p, rescue assays were performed to investigate whether LINC01535 exerted its role in osteogenic differentiation via the miR-3619-5p/BMP2 axis. Following transfection with miR-3619 inhibitor and sh-BMP2, the expression levels of miR-3619-5p and BMP2 were significantly downregulated compared with their respective NCs (Fig. 5A). Furthermore, RT-qPCR and western blotting revealed that the downregulated mRNA and protein expression levels of OCN, OSX and RUNX2 caused by LINC01535 silencing increased following miR-3619-5p suppression, while the expression was partially recovered following BMP2 depletion (Fig. 5B and C).
The ALP activity assay further validated that miR-3619-5p inhibitor reversed the regulatory impact of LINC01535 knockdown on ALP activity, while the restoration of osteogenic differentiation occurred following BMP2 downregulation (Fig. 5D). Moreover, miR-3619-5p inhibition significantly increased the cell viability repressed by LINC01535 inhibition, while the silencing of BMP2 restored the viability of hBMSCs at $72 \mathrm{~h}$ (Fig. 5E). These observations suggested that LINC01535 may function as a facilitator in osteogenesis via modulating the miR-3619-5p/BMP2 axis.

\section{Discussion}

Osteoporosis is a common type of bone metabolic disorder disease that is attributed to the excess of bone absorption over bone formation $(33,34)$. The characteristics of osteoporosis include diminished bone mineral density, bone loss, microarchitecture destruction and increased risks of fractures, ultimately leading to bone collapse and fracture $(35,36)$. Despite significant progress being made in the treatment of osteoporosis, the underlying mechanisms of osteoporosis 
remain poorly understood (37). BMSCs can differentiate into osteoblasts, which neutralize bone absorption mediated by osteoclasts (38). Accordingly, identifying regulators of osteogenic differentiation in BMSCs is of significance to help shed light on the pathogenesis of osteoporosis.

An increasing number of investigations have reported that $\operatorname{lncRNAs}$ regulate a multitude of cellular processes that participate in a diverse range of diseases, including osteoporosis $(29,39,40)$. In fact, emerging evidence has identified that lncRNAs participated in osteoporosis progression (41-43). For example, the suppression of IncRNA anti-differentiation noncoding RNA facilitated the osteogenesis of osteoblasts in postmenopausal osteoporosis via modulating histone-lysine N-methyltransferase EZH2 and RUNX2 (44); the IncRNA X inactive specific transcript enhanced osteoporosis via repressing bone marrow mesenchymal stem cell differentiation (45) and the IncRNA KCNQ1OT1 upregulated BMP2 expression levels to promote osteogenic differentiation by competitively binding to miR-214 (31). It was also shown that LINC01535 functioned as an oncogene in the development of cervical cancer (21). However, to the best of our knowledge, there are very few investigations into the role and potential mechanism of LINC01535 in the osteogenic differentiation of BMSCs. The findings of the present study revealed that LINC01535 was highly expressed during osteogenic induction. Further functional assays indicated that the silencing of LINC01535 attenuated the growth and osteogenic differentiation of hBMSCs.

lncRNAs serve as natural sponges of miRNAs, which subsequently interferes with downstream target genes (46-48). Therefore, the present study hypothesized that LINC01535 may exert its function in osteogenic differentiation by acting as a sponge for a specific miRNA. Using the starBase website, a potential miR-3619-5p binding site was identified in the 3'-UTR of LINC01535. A luciferase reporter assay subsequently validated the interaction between LINC01535 and miR-3619-5p. Previous studies reported that lncRNAs could regulate the progression of various cancers, such as papillary thyroid carcinoma (49), hemangioma (50) and retinoblastoma (51) by targeting miR-3619. Furthermore, BMP2, as a crucial modulator of osteogenic differentiation, was confirmed to be a target of miR-3619-5p. BMP2 has been reported to be involved in osteogenic differentiation. Jiang et al (52) indicated that miR-204 suppressed the osteogenic differentiation of mesenchymal stem cells by regulating BMP2. Furthermore, Zhang et al (53) demonstrated that overexpression of miR-93-5p inhibited osteogenic differentiation by downregulating BMP2. In the present study, the results suggested that LINC01535 may affect the expression levels of BMP2 via sponging miR-3619-5p. Ultimately, these findings verified that LINC01535 promoted BMP2 expression to exert its promoting role in the osteogenic regulation of hBMSCs by competing for miR-3619-5p.

To the best of our knowledge, the present study was the first to characterize the expressional pattern and regulatory function of LINC01535 during the osteogenic differentiation process. The results suggested that LINC01535 may accelerate the osteogenic differentiation of hBMSCs via targeting the miR-3619-5p/BMP2 pathway, which provided an improved understanding of the pathogenesis of osteoporosis and suggested that the LINC01535/miR-3619-5p/BMP2 network may represent a latent therapeutic strategy for the treatment of osteoporosis. However, the present study has several limitations, which remain to be addressed; for example, the findings were obtained from in vitro assays, thus, the role of the LINC01535/miR-3619-5p/BMP2 axis should be further investigated in vivo.

\section{Acknowledgements}

Not applicable.

\section{Funding}

No funding was received.

\section{Availability of data and materials}

The datasets used and/or analyzed during the current study are available from the corresponding author on reasonable request.

\section{Authors' contributions}

YZ and FW designed the present study. YC and XH performed all the experiments. $\mathrm{NZ}$ and $\mathrm{XH}$ analyzed the data and prepared the figures. YZ drafted the initial manuscript. FW reviewed and revised the manuscript. All authors read and approved the final manuscript.

\section{Ethics approval and consent to participate}

Not applicable.

\section{Patient consent for publication}

Not applicable.

\section{Competing interests}

The authors declare that they have no competing interests.

\section{References}

1. Feng $\mathrm{X}$ and McDonald JM: Disorders of bone remodeling. Annu Rev Pathol 6: 121-145, 2011.

2. Mulari $M T, Q u$ Q, Härkönen $\mathrm{PL}$ and Väänänen $\mathrm{HK}$ : Osteoblast-like cells complete osteoclastic bone resorption and form new mineralized bone matrix in vitro. Calcif Tissue Int 75: 253-261, 2004.

3. Bennett CN, Longo KA, Wright WS, Suva LJ, Lane TF, Hankenson KD and MacDougald OA: Regulation of osteoblastogenesis and bone mass by Wnt10b. Proc Natl Acad Sci USA 102: 3324-3329, 2005.

4. Chen P, Li Z and Hu Y: Prevalence of osteoporosis in China: A meta-analysis and systematic review. BMC Public Health 16: 1039, 2016.

5. Phan TC, Xu J and Zheng MH: Interaction between osteoblast and osteoclast: Impact in bone disease. Histol Histopathol 19: 1325-1344, 2004.

6. Pittenger MF, Mackay AM, Beck SC, Jaiswal RK, Douglas R, Mosca JD, Moorman MA, Simonetti DW, Craig S and Marshak DR: Multilineage potential of adult human mesenchymal stem cells. Science 284: 143-147, 1999.

7. Bruder SP, Kurth AA, Shea M, Hayes WC, Jaiswal N and Kadiyala S: Bone regeneration by implantation of purified, culture-expanded human mesenchymal stem cells. J Orthop Res 16: 155-162, 1998. 
8. Zhang W, Yang GJ, Wu SX, Li DQ, Xu YB, Ma CH, Wang JL and Chen WW: The guiding role of bone metabolism test in osteoporosis treatment. Am J Clin Exp Immunol 7: 40-49, 2018.

9. Xiong L, Jung JU, Wu H, Xia WF, Pan JX, Shen C, Mei L and Xiong WC: Lrp4 in osteoblasts suppresses bone formation and promotes osteoclastogenesis and bone resorption. Proc Natl Acad Sci USA 112: 3487-3492, 2015.

10. Quinn JJ and Chang HY: Unique features of long non-coding RNA biogenesis and function. Nat Rev Genet 17: 47-62, 2016.

11. Iyer MK, Niknafs YS, Malik R, Singhal U, Sahu A, Hosono Y, Barrette TR, Prensner JR, Evans JR, Zhao S, et al: The landscape of long noncoding RNAs in the human transcriptome. Nat Genet 47: 199-208, 2015.

12. Josefs $\mathrm{T}$ and Boon RA: The long non-coding road to atherosclerosis. Curr Atheroscler Rep 22: 55, 2020.

13. Ghafouri-Fard S, Eghtedarian R and Taheri M: The crucial role of non-coding RNAs in the pathophysiology of inflammatory bowel disease. Biomed Pharmacother 129: 110507, 2020.

14. Chen W, Yang J, Fang H, Li L and Sun J: Relevance function of linc-ROR in the pathogenesis of cancer. Front Cell Dev Biol 8: 696, 2020.

15. Guttman M, Donaghey J, Carey BW, Garber M, Grenier JK, Munson G, Young G, Lucas AB, Ach R, Bruhn L, et al: lincRNAs act in the circuitry controlling pluripotency and differentiation. Nature 477: 295-300, 2011.

16. Wang CG, Hu YH, Su SL and Zhong D: lncRNA DANCR and miR-320a suppressed osteogenic differentiation in osteoporosis by directly inhibiting the Wnt/ $\beta$-catenin signaling pathway. Exp Mol Med 52: 1310-1325, 2020.

17. Ma J, Zhang X, Zhang H and Chen H: lncRNA MEG3 suppresses the progression of ankylosis spondylitis by regulating the Let-7i/SOST axis. Front Mol Biosci 7: 173, 2020.

18. Zheng S, Wang YB, Yang YL, Chen BP, Wang CX, Li RH and Huang D: lncRNA MALAT1 inhibits osteogenic differentiation of mesenchymal stem cells in osteoporosis rats through MAPK signaling pathway. Eur Rev Med Pharmacol Sci 23: 4609-4617, 2019.

19. Zhuang W, Ge X, Yang S, Huang M, Zhuang W, Chen P, Zhang X, Fu J, Qu J and Li B: Upregulation of lncRNA MEG3 promotes osteogenic differentiation of mesenchymal stem cells from multiple myeloma patients by targeting BMP4 transcription. Stem Cells 33: 1985-1997, 2015.

20. Wei B, Wei W, Zhao B, Guo X and Liu S: Long non-coding RNA HOTAIR inhibits miR-17-5p to regulate osteogenic differentiation and proliferation in non-traumatic osteonecrosis of femoral head. PLoS One 12: e0169097, 2017.

21. Song H, Liu Y, Jin X, Liu Y, Yang Y, Li L, Wang X and Li G: Long non-coding RNA LINC01535 promotes cervical cancer progression via targeting the miR-214/EZH2 feedback loop. J Cell Mol Med 23: 6098-6111, 2019.

22. Carthew RW and Sontheimer EJ: Origins and mechanisms of miRNAs and siRNAs. Cell 136: 642-655, 2009.

23. Rana TM: Illuminating the silence: Understanding the structure and function of small RNAs. Nat Rev Mol Cell Biol 8: 23-36, 2007.

24. Garzon R, Calin GA and Croce CM: MicroRNAs in cancer Annu Rev Med 60: 167-179, 2009

25. An JH, Ohn JH, Song JA, Yang JY, Park H, Choi HJ, Kim SW, Kim SY, Park WY and Shin CS: Changes of microRNA profile and microRNA-mRNA regulatory network in bones of ovariectomized mice. J Bone Miner Res 29: 644-656, 2014.

26. Zhang H, Hua Y, Jiang Z, Yue J, Shi M, Zhen X, Zhang X, Yang L, Zhou R and Wu S: Cancer-associated fibroblast-promoted lncRNA DNM3OS confers radioresistance by regulating DNA damage response in esophageal squamous cell carcinoma. Clin Cancer Res 25: 1989-2000, 2019.

27. Kim J, Kim IS, Cho TH, Lee KB, Hwang SJ, Tae G, Noh I, Lee SH, Park Y and Sun K: Bone regeneration using hyaluronic acid-based hydrogel with bone morphogenic protein-2 and human mesenchymal stem cells. Biomaterials 28: 1830-1837, 2007.

28. Shu B, Zhang M, Xie R, Wang M, Jin H, Hou W, Tang D, Harris SE, Mishina Y, O'Keefe RJ, et al: BMP2, but not BMP4, is crucial for chondrocyte proliferation and maturation during endochondral bone development. J Cell Sci 124: 3428-3440, 2001.

29. Zhang N, Hu X, He S, Ding W, Wang F, Zhao Y and Huang Z lncRNA MSC-AS1 promotes osteogenic differentiation and alleviates osteoporosis through sponging microRNA-140-5p to upregulate BMP2. Biochem Biophys Res Commun 519: 790-796, 2019 .
30. Qiao L, Li CG and Liu D: CircRNA 0048211 protects postmenopausal osteoporosis through targeting miRNA-93-5p to regulate BMP2. Eur Rev Med Pharmacol Sci 24: 3459-3466, 2020.

31. Wang CG, Liao Z, Xiao H, Liu H, Hu YH, Liao QD and Zhong D: lncRNA KCNQ1OT1 promoted BMP2 expression to regulate osteogenic differentiation by sponging miRNA-214. Exp Mol Pathol 107: 77-84, 2019.

32. Livak KJ and Schmittgen TD: Analysis of relative gene expression data using real-time quantitative PCR and the 2(-Delta Delta C(T)) method. Methods 25: 402-408, 2001.

33. Ahmed SF and Elmantaser M: Secondary osteoporosis. Endocr Dev 16: 170-190, 2009.

34. Teitelbaum SL: Bone resorption by osteoclasts. Science 289 : 1504-1508, 2000

35. Li WF, Hou SX, Yu B, Li MM, Férec C and Chen JM: Genetics of osteoporosis: Accelerating pace in gene identification and validation. Hum Genet 127: 249-285, 2010.

36. Tu KN, Lie JD, Wan CKV, Cameron M, Austel AG, Nguyen JK, Van K and Hyun D: Osteoporosis: A review of treatment options. P T 43: 92-104, 2018.

37. Adler RA: Osteoporosis treatment: Complexities and challenges. J Endocrinol Invest 39: 719-720, 2016.

38. Clarke B: Normal bone anatomy and physiology. Clin J Am Soc Nephrol 3 (Suppl 3): S131-S139, 2008.

39. Liang C, Zhao T, Li H, He F, Zhao X, Zhang Y, Chu X, Hua C, $\mathrm{Qu}$ Y, Duan Y, et al: Long non-coding RNA ITIH4-AS1 accelerates the proliferation and metastasis of colorectal cancer by activating JAK/STAT3 signaling. Mol Ther Nucleic Acids 18: 183-193, 2019.

40. Li FP, Ling DQ and Gao LY: Long noncoding RNA Chaer mediated Polycomb Repressor Complex 2 (PRC2) activity to promote atherosclerosis through mTOR signaling. Eur Rev Med Pharmacol Sci 23: 7639-7648, 2019.

41. Mei B, Wang Y, Ye W, Huang H, Zhou Q, Chen Y, Niu Y, Zhang $M$ and Huang Q: lncRNA ZBTB40-IT1 modulated by osteoporosis GWAS risk SNPs suppresses osteogenesis. Hum Genet 138: 151-166, 2019.

42. Shen JJ, Zhang CH, Chen ZW, Wang ZX, Yang DC, Zhang FL and Feng KH: IncRNA HOTAIR inhibited osteogenic differentiation of BMSCs by regulating Wnt// -catenin pathway. Eur Rev Med Pharmacol Sci 23: 7232-7246, 2019.

43. Zhang RF, Liu JW, Yu SP, Sun D, Wang XH, Fu JS and Xie Z: lncRNA UCA1 affects osteoblast proliferation and differentiation by regulating BMP-2 expression. Eur Rev Med Pharmacol Sci 23: 6774-6782, 2019.

44. Cai N, Li C and Wang F: Silencing of lncRNA-ANCR promotes the osteogenesis of osteoblast cells in postmenopausal osteoporosis via targeting EZH2 and RUNX2. Yonsei Med J 60: 751-759, 2019.

45. Chen X, Yang L, Ge D, Wang W, Yin Z, Yan J, Cao X, Jiang C, Zheng S and Liang B: Long non-coding RNA XIST promotes osteoporosis through inhibiting bone marrow mesenchymal stem cell differentiation. Exp Ther Med 17: 803-811, 2019.

46. Salmena L, Poliseno L, Tay Y, Kats L and Pandolfi PP: A ceRNA hypothesis: The rosetta stone of a hidden RNA language? Cell 146: 353-358, 2011

47. Sun F, Liang W, Tang K, Hong $M$ and Qian J: Profiling the lncRNA-miRNA-mRNA ceRNA network to reveal potential crosstalk between inflammatory bowel disease and colorectal cancer. PeerJ 7: e7451, 2019

48. Zhou RS, Zhang EX, Sun QF, Ye ZJ, Liu JW, Zhou DH and Tang Y: Integrated analysis of IncRNA-miRNA-mRNA ceRNA network in squamous cell carcinoma of tongue. BMC Cancer 19: 779, 2019.

49. Wang G, Wang X and Jin Y: LINC01410/miR-3619-5p/FOXM1 feedback loop regulates papillary thyroid carcinoma cell proliferation and apoptosis. Cancer Biother Radiopharm 34: 572-580, 2019.

50. Liu Z, Kang Z, Dai Y, Zheng $\mathrm{H}$ and Wang Y: Long noncoding RNA LINC00342 promotes growth of infantile hemangioma by sponging miR-3619-5p from HDGF. Am J Physiol Heart Circ Physiol 317: H830-H839, 2019.

51. Yan G, Su Y, Ma Z, Yu L and Chen N: Long noncoding RNA LINC00202 promotes tumor progression by sponging miR-3619-5p in retinoblastoma. Cell Struct Funct 44: 51-60, 2019.

52. Jiang X, Zhang Z, Peng T, Wang G, Xu Q and Li G: miR-204 inhibits the osteogenic differentiation of mesenchymal stem cells by targeting bone morphogenetic protein 2. Mol Med Rep 21: $43-50,2020$

53. Zhang Y, Wei QS, Ding WB, Zhang LL, Wang HC, Zhu YJ, He W, Chai YN and Liu YW: Increased microRNA-93-5p inhibits osteogenic differentiation by targeting bone morphogenetic protein-2. PLoS One 12: e0182678, 2017. 